\title{
Collapse and expansion in the bright-rimmed cloud SFO 11NE
}

\author{
M. A. Thompson and G. J. White
}

Centre for Astrophysics \& Planetary Science, School of Physical Sciences, University of Kent, Canterbury, Kent CT2 7NR, UK

Received 8 December 2003 / Accepted 19 February 2004

\begin{abstract}
We report the results of a search for the double-peaked blue-skewed infall signature in the bright-rimmed cloud core SFO $11 \mathrm{NE}$ SMM1. Observations of the optically thick $\mathrm{HCO}^{+}$and optically thin $\mathrm{H}^{13} \mathrm{CO}^{+} J=3-2$ lines reveal that there is indeed a characteristic double-peaked line profile, but skewed to the red rather than the blue. Modelling of the dust continuum emission and line profiles show that the motions within SFO 11NE SMM1 are consistent with a collapsing central core surrounded by an expanding outer envelope. We show that the collapse is occurring at a similar rate to that expected onto a single solar-mass protostar and is unlikely to represent the large-scale collapse of gas onto the infrared cluster seen at the heart of SFO 11NE SMM1. The outer envelope is expanding at a much greater rate than that expected for a photoevaporated flow from the cloud surface. The modelled expansion is consistent with the bulk cloud re-expansion phase predicted by radiative-driven implosion models of cometary clouds.
\end{abstract}

Key words. stars: formation - ISM: individual object: SFO 11NE - ISM: clouds - ISM: dust, extinction - ISM: molecules submillimeter

\section{Introduction}

Spectroscopic evidence for collapse motions in low-mass starforming regions has now become relatively widespread (e.g. Myers et al. 2000; Evans 1999). The classic spectroscopic signature of infall or collapse is an asymmetric blue-skewed double-peaked optically thick line profile with self-absorption at the systemic velocity (Myers et al. 2000). The interpretation of this profile can be fraught with difficulty: unrelated clouds located along the line-of-sight can mimic the infall signature (Myers et al. 2000), molecular outflows add extra complexity to the gas kinematics (Hogerheijde et al. 1998), chemical effects such as depletion may mask the infall signature completely (Rawlings \& Yates 2001) and rotation of the molecular core can completely reverse the blue-skewed profile to the red (Zhou 1995). In order to conclusively demonstrate the presence of infall it is usually necessary to map the emission of both optically thick and thin lines across the molecular cloud core and compare their profiles to collapse models (e.g. Gregersen et al. 2000; Lee et al. 2001).

Despite these difficulties, collapse motions have been identified in several protostellar and preprotostellar cores. The objects that have been mainly investigated so far are Class 0 \& I protostars (Gregersen et al. 1997; Mardones et al. 1997) and preprotostellar cores (Lee et al. 1999, 2001). Most of these objects are located in nearby relatively isolated low-mass star-forming regions such as the Perseus, Serpens, Taurus and Ophiuchus molecular clouds (Mardones et al. 1997). Few

Send offprint requests to: M. A. Thompson,

e-mail: m.a.thompson@kent.ac.uk star-forming regions associated with the clustered star-forming mode (Lada et al. 1993) have been searched for the presence of infall. Two notable exceptions include recent searches for collapse motions of gas associated with young stellar clusters themselves (Williams \& Myers 1999; Williams \& Garland 2002) and for infall within bright-rimmed clouds associated with large HII regions (de Vries et al. 2002).

Both types of search report limited success with collapse motions identified in two young clusters (Cepheus A and NGC 2264) and one bright-rimmed cloud (SFO 18). It is the latter type of object that we will focus on in this paper. Brightrimmed clouds are potential regions of triggered or induced star formation (Sugitani et al. 1991; Sugitani \& Ogura 1994), whereby the collapse of the molecular gas comprising the cloud and the ensuing star formation process is initiated by the action of an external trigger. In bright-rimmed clouds the external trigger is most likely the photoionisation-induced shocks that are driven into the clouds by the ionisation of their outer layers by nearby OB stars (e.g. Elmegreen 1991). These shocks compress the molecular gas of the clouds, triggering the collapse of the cloud and forming a dense, possibly quasistatic core at the cloud centre (Bertoldi 1989; Lefloch \& Lazareff 1994, 1995).

However, although de Vries, Narayanan \& Snell (2002) report the detection of an infall signature towards the bright-rimmed cloud SFO 18, it is the exception rather than the rule in their sample of bright-rimmed clouds. The overall blue excess of the bright-rimmed cloud sample is less than that of the Class $0 \&$ I protostellar cores observed by Mardones et al. (1997) and Gregersen et al. (2000). This phenomenon is puzzling given the strong velocity gradients predicted by the 
Lefloch \& Lazareff (1994) radiative-driven implosion model. However the small sample size of de Vries et al. (2002) means that their result may not be statistically significant. It is also possible that a flattened temperature gradient across the brightrimmed clouds, caused by their external heating, could mask the classic asymmetric infall signature (de Vries el al. 2002). A wider infall survey of a larger number of bright-rimmed clouds, coupled with more realistic radiative transfer modelling of their temperature and velocity gradients is required to address the issue of infall in these clouds. Such a study would also permit the detailed investigation of the kinematics of these clouds, which is important in the context of refining and expanding the existing radiative driven implosion models to take into account star formation processes (e.g. Bertoldi 1989; Lefloch \& Lazareff 1994, 1995).

We are currently undertaking such a survey of a statistically significant sample of bright-rimmed clouds. As a fore-runner to the survey we present the results of a search for infall toward the bright-rimmed cloud SFO $11 \mathrm{NE}$. SFO $11 \mathrm{NE}$ is located at the edge of the HII region IC 1848, approximately 4' NE of the bright-rimmed cloud SFO 11 (Thompson et al. 2004; Sugitani et al. 2002). SCUBA sub-mm continuum and JCMT CO observations reveal a dense core of molecular gas and dust located at the head of the cometary cloud (SFO 11NE SMM1), whilst 2MASS images show that the dense dust core harbours a cluster of candidate young stellar objects and protostars (Thompson et al. 2004). The pressure balance of the cloud could suggest that the cloud is likely to be in the early collapse phase described by Lefloch \& Lazareff (1994) and is thus a good candidate in which to search for infall.

\section{Observational procedure and results}

We obtained five spectra of the $\mathrm{HCO}^{+} J=3-2$ line at 267.5576 $\mathrm{GHz}$ using the facility $A$-band heterodyne receiver at the James Clerk Maxwell Telescope $\left(\mathrm{JCMT}^{1}\right.$ ) during June 2002. The spectra were arranged in a 5-point cross-shaped grid centred upon the sub-mm continuum peak of SFO 11NE SMM1 $\left(\alpha(2000)=02^{\mathrm{h}} 51^{\mathrm{m}} 53^{\mathrm{s}} \cdot 6, \delta(2000)=+60^{\circ} 07^{\prime} 00^{\prime \prime}\right)$. The $F W H M$ beamwidth of the JCMT is $\sim 22^{\prime \prime}$ at this frequency and the cross-shaped grid was spaced by $15^{\prime \prime}$. A single spectrum of the $\mathrm{H}^{13} \mathrm{CO}^{+} J=3-2$ line at $260.2555 \mathrm{GHz}$ at the sub-mm continuum peak position was also obtained. The $\mathrm{HCO}^{+}$line is usually optically thick towards typical star-forming regions (e.g. Gregersen et al. 2000), whereas the less common isotopomeric line is usually optically thin.

All spectra were obtained using frequency-switched mode with a local oscillator switching offset of $8.2 \mathrm{MHz}$ backend spectrometer was set to its minimum bandwidth of $125 \mathrm{MHz}$ to obtain the maximum possible velocity resolution of $\sim 0.1 \mathrm{~km} \mathrm{~s}^{-1}$ per $94 \mathrm{kHz}$ wide channel. The integration times for the $\mathrm{HCO}^{+}$spectra and $\mathrm{H}^{13} \mathrm{CO}^{+}$spectrum were $20 \mathrm{~min}$ and 40 min respectively, which resulted in an rms noise per $0.1 \mathrm{~km} \mathrm{~s}^{-1}$ wide channel of 90 and $68 \mathrm{mK}$.

\footnotetext{
1 The JCMT is operated by the Joint Astronomy Centre on behalf of PPARC for the United Kingdom, the Netherlands Organisation of Scientific Research, and the National Research Council of Canada.
}

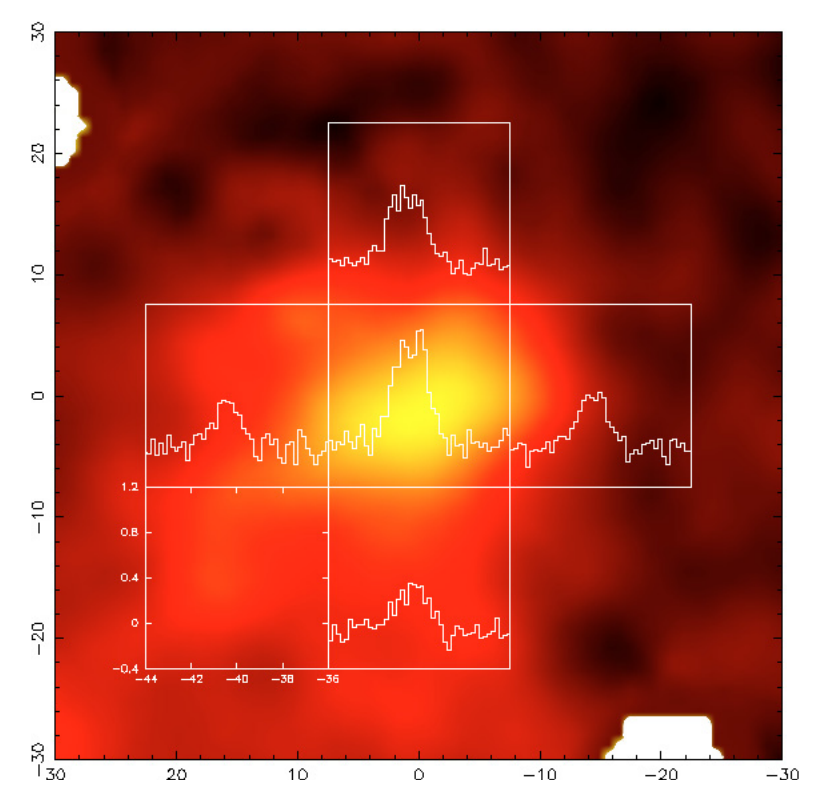

Fig. 1. Grid-map of the $\mathrm{HCO}^{+} J=3-2$ spectra overlaid on an $850 \mu \mathrm{m}$ SCUBA image of SFO 11NE SMM1 (Thompson et al. 2004). The map centre is at $\alpha(2000)=02^{\mathrm{h}} 51^{\mathrm{m}} 53^{\mathrm{s}} .6, \delta(2000)=+60^{\circ} 07^{\prime} 00^{\prime \prime}$.

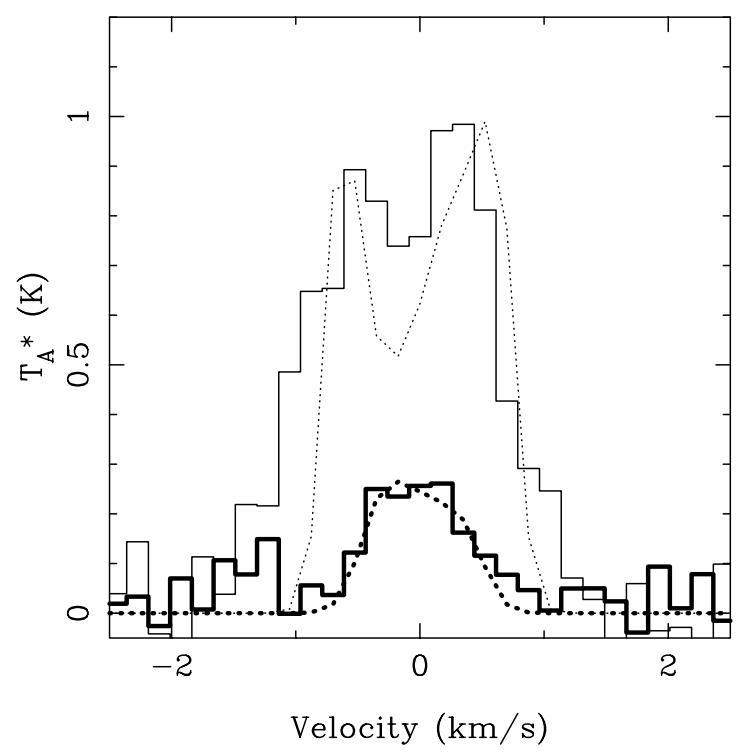

Fig. 2. Composite spectrum of the $\mathrm{HCO}^{+}$(thin) and the $\mathrm{H}^{13} \mathrm{CO}^{+}$ (thick) $J=3-2$ lines observed toward the sub-mm continuum peak of SFO 11NE SMM1. Model spectra from the best fitting line model described in Sect. 3 are shown by dotted thin and thick lines.

The data were reduced using the Starlink package SPECX (Prestage et al. 2000). To improve the signal-to-noise ratio the spectra were binned to a velocity resolution of $0.2 \mathrm{~km} \mathrm{~s}^{-1}$. The $\mathrm{HCO}^{+}$spectra are shown in Fig. 1, overlaid on the SCUBA $850 \mu \mathrm{m}$ image from Thompson et al. (2004). The central $\mathrm{HCO}^{+}$spectrum clearly displays a double-peaked profile in both the binned and unbinned spectra, although skewed to the red rather than the typical blue-excess infall profile (Myers et al. 2000). A composite spectrum of the $\mathrm{HCO}^{+}$and $\mathrm{H}^{13} \mathrm{CO}^{+}$spectra taken at the central position may be found in Fig. 2. The $\mathrm{H}^{13} \mathrm{CO}^{+}$line has a moderate asymmetry, with a clearly visible red wing. 


\section{Modelling the dust continuum emission and gas kinematics}

The overall appearance of the $\mathrm{HCO}^{+}$emission is puzzling. At the central position of the core the $\mathrm{HCO}^{+}$emission is doublepeaked, which is suggestive of infall, but the emission from the red peak is greater than that of the blue, perhaps implying that the core is expanding rather than collapsing. The optically thin $\mathrm{H}^{13} \mathrm{CO}^{+}$emission is singly peaked with a central velocity at that of the self-absorption dip, ruling out the possibility that the shape of the $\mathrm{HCO}^{+}$emission is caused by two unrelated line-of-sight molecular clumps. Away from the central position the $\mathrm{HCO}^{+}$spectra are more symmetric, without clearly visible absorption dips. The outer spectra are also approximately the same brightness, although the southern and western spectra are slightly weaker than the remaining two spectra, which is perhaps due to the fact that the molecular cloud is sharply truncated in these directions (Thompson et al. 2004). The east and west spectra appear to be slightly velocity-shifted from one another, which may suggest that the core rotates about its northsouth axis, although in these cases the emission toward the centre of the core is usually symmetric rather than skewed to the red (Zhou 1995).

As mentioned in the Introduction, the interpretation of infall line profiles can be fraught with difficulty and in order to understand the kinematics of the molecular gas comprising SFO 11NE SMM1 we carried out detailed radiative transfer modelling of the sub-mm continuum and $\mathrm{HCO}^{+} / \mathrm{H}^{13} \mathrm{CO}^{+}$emission. As SFO 11NE SMM1 is only marginally elongated along the NW-SE axis in the SCUBA image and the line emission suggests that the core is reasonably spherical with no strong velocity gradient we decided to model the emission using the RATRAN one-dimensional radiative transfer code (Hogerheijde \& van der Tak 2000).

RATRAN solves for the line and dust continuum emission simultaneously. Firstly, we used the azimuthally averaged SCUBA $850 \mu \mathrm{m}$ profile and the spectral energy distribution (at 60,450 and $850 \mu \mathrm{m}$ ) published by Thompson et al. (2004) to fix the size, temperature and density profile of SFO 11NE SMM1. In the modelling described in this paper we assumed the dust opacity model of Ossenkopf \& Henning (1994) with thick ice mantles and $10^{5}$ years of grain growth. Our physical model for SFO 11NE SMM1 is that of a spherical core of radius $8 \times 10^{17} \mathrm{~cm}(0.26 \mathrm{pc})$ comprised of 10 logarithmically spaced radially symmetric shells with an inner cutoff at $5 \times 10^{16} \mathrm{~cm}$. Given that we possess information on the spatial distribution of the dust emission at only one wavelength $(850 \mu \mathrm{m})$ we did not feel it was appropriate to model the density power-law index as an additional parameter and so we assumed a density profile $r^{-3 / 2}$.

We systematically explored a wide range of parameter space to find the best model fit to the data, including models with inwardly-increasing, outwardly-increasing and flat (i.e. constant) temperature gradients. In all models we assumed that the gas and dust temperatures were equal, as is expected for high-density star-forming cores. The model flux profile was convolved by a $14^{\prime \prime} F W H M$ beam to match the SCUBA observations. The best fitting model and the observed azimuthally
Radial $850 \mu \mathrm{m}$ flux profile

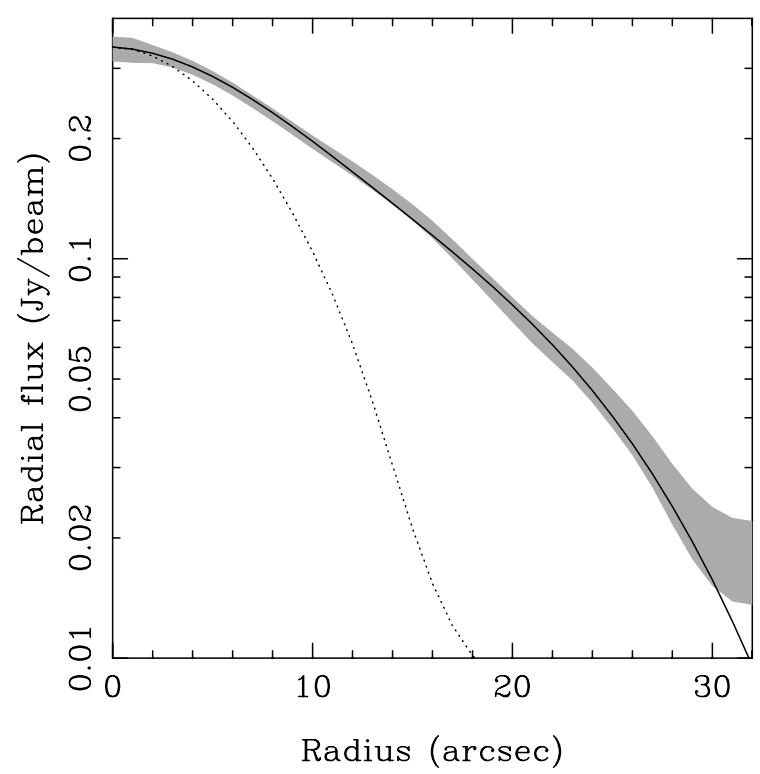

Fig. 3. The azimuthally averaged $850 \mu \mathrm{m}$ flux profile and the best model fit to the profile. The $850 \mu \mathrm{m}$ flux profile and its uncertainty is represented by the shaded area. The best model fit is shown by a solid line and the JCMT beam profile is represented by the dotted line.

averaged $850 \mu \mathrm{m}$ flux profile are shown in Fig. 3. Figure 3 also shows that there is an upturn in the $850 \mu \mathrm{m}$ flux at large radii $\left(>30^{\prime \prime}\right)$, which is more than likely due to the influence of the neighbouring core SFO 11NE SMM2 lying 50" to the southeast (Thompson et al. 2004).

We could not reproduce either the observed spectral energy distribution (SED) or the azimuthally averaged flux profile with a single-temperature model nor with an outwardlyincreasing temperature gradient. An outwardly-increasing temperature gradient results in a much flatter flux profile than is observed, as the outer layers start to contribute more towards the total sub-mm flux. If the dust at the outer edge of the core is heated by the UV radiation from the nearby O-star it must comprise a relatively thin and low-mass layer that does not substantially contribute to the $850 \mu \mathrm{m}$ emission. By experimentation we found that the best fits to the SED and flux profile were obtained by using a two temperature model with the inner three shells at $30 \mathrm{~K}$ and the remaining outer shells at $18 \mathrm{~K}$.

The best-fitting density profile for the two temperature model was found to follow the form $n_{\mathrm{H}_{2}}(r)=4.5 \times$ $10^{3}\left(r_{\max } / r\right)^{3 / 2}$. Both the temperature and density compare well with the values derived from the single-component greybody model of Thompson et al. (2004). The observed flux profile and SED are reproduced well by our two-temperature $r^{-3 / 2}$ density profile model. The observed 60 and $450 \mu \mathrm{m}$ integrated fluxes are reproduced to within $20 \%$ and the $850 \mu \mathrm{m}$ integrated flux to within $10 \%$. We estimated the sensitivity of the best-fitting model parameters to the data by progressively modifying the model parameters away from the best-fitting values. We find that the temperature (for each component) must lie within $2 \mathrm{~K}$ and that the outer density must lie within $3 \times 10^{2} \mathrm{~cm}^{-3}$ of the best-fitting value. 
Using the dust continuum emission to fix the size of the core and its temperature and density profiles allows us to constrain the model of the line emission very closely. The only free parameters in our subsequent modelling of the line emission are the abundance of $\mathrm{HCO}^{+}$, the mean velocity of each shell and the velocity dispersion of the molecular gas. It is also possible to treat the ${ }^{12} \mathrm{C} /{ }^{13} \mathrm{C}$ abundance ratio as another free parameter but in the modelling we carried out for this paper we assumed a standard ${ }^{12} \mathrm{C} /{ }^{13} \mathrm{C}$ abundance ratio of 60 (Frerking et al. 1982).

We modelled the $\mathrm{HCO}^{+}$and $\mathrm{H}^{13} \mathrm{CO}^{+}$emission from SFO 11NE SMM1 along three broad hypotheses: pure infall, pure expansion, or a mixture of infall and expansion. The former set of models were included as a cross-check of our method as models comprised of pure infall should be unable to reproduce the observed red-skewed profile. The redskewed $\mathrm{HCO}^{+}$line profile suggests the presence of expanding gas which may originate from either bulk expansion of the gas comprising the core or molecular outflows driven by protostars embedded within the core. There is some evidence that molecular outflows are associated with SFO 11NE SMM1, by the presence of moderate velocity $\mathrm{CO}$ line wings and a possible photoionised jet (Thompson et al. 2004), but no other supporting evidence (e.g. spatially separated outflow lobes) has yet been found. Without spatially-resolved outflow lobes it is not possible to model the gas expansion in terms of a molecular outflow and so in all the expansion models described in this paper the expansion is modelled as a bulk expansion of the core. Nevertheless it is worth noting that any gas expansion inferred by the modelling may result from molecular outflows rather than a bulk expansion of the core. We will return to this point in Sect. 4

As expected, it was found that models comprised of pure infalling gas could not match the observed red-excess emission along the central line-of-sight nor the apparent symmetry of the outer lines-of-sight. Models consisting of a purely expanding core could not reproduce the level of asymmetry between the red and blue peaks of the central line-of-sight spectrum. In this latter case the blue peak is always much less bright than the red peak. For both sets of models we investigated whether velocity gradients across the model core (e.g. increasing infall velocity of each shell with decreasing shell radius or vice versa) could match the observed line profiles. In all cases the observed $\mathrm{HCO}^{+}$emission could not be reproduced by either pure infall or pure expansion.

We decided to restrict the models including a mixture of infalling and expanding gas to a simple two-layer interpretation to simplify the modelling process and to reduce the number of free parameters in the model. This two-layer interpretation is similar to the simplified model described by Myers et al. (2000) but with spherical geometry rather than two uniform collapsing layers. For simplicity we will refer to the central portions of our spherical model as the "core" and the surrounding outer layers as the "envelope". We considered cases for both an expanding core combined with a collapsing envelope (e.g. NGC 2264 IRS1; Williams \& Garland 2002); and a collapsing core combined with an expanding envelope. In addition we modelled the subsets of these two cases with either static cores or envelopes.
The best fit to the observed $\mathrm{HCO}^{+}$and $\mathrm{H}^{13} \mathrm{CO}^{+}$spectra (i.e. with the lowest residuals) was produced by a model comprised of a collapsing core surrounded by an expanding envelope. Models consisting of an expanding core surrounded by a collapsing envelope produce a central blue-skewed profile and are not consistent with the observed spectra. The limited number of free parameters in the model $\left(\mathrm{HCO}^{+}\right.$abundance profile, mean velocity and velocity dispersion) and the sharp discontinuity between the two layers meant that it was difficult to closely reproduce the depth of the $\mathrm{HCO}^{+}$absorption dip between the red and blue peaks of the central spectrum. Nevertheless, the collapsing core and expanding envelope model closely matches the relative brightness of both peaks, their displacement from one another in velocity, the moderate red asymmetry of the $\mathrm{H}^{13} \mathrm{CO}^{+}$line and the relative symmetry of the outer spectra.

We could not simultaneously fit any of the outlying $\mathrm{HCO}^{+}$spectra in the grid-map with a single model and this probably reflects the limitations of assuming spherical symmetry in the model. However, the collapsing core and expanding envelope model produces the closest fit to the outer spectra of all modelled hypotheses. The models comprised of pure infall or pure expansion show pronounced asymmetry in their outer $\mathrm{HCO}^{+}$spectra. Only by combining infalling and expanding gas motions in the model can we reduce the asymmetry in the outer $\mathrm{HCO}^{+}$model spectra to approach that seen in the observed outer $\mathrm{HCO}^{+}$spectra. Also, given the close match of the model to the $\mathrm{HCO}^{+}$and $\mathrm{H}^{13} \mathrm{CO}^{+}$spectra along the central lineof-sight we are encouraged that our model interpretation is a good representation of the gas motions within the core.

The largest discrepancy is in the spectrum to the north of SFO 11NE SMM1, which is much broader and brighter than the model suggests. The eastern and western $\mathrm{HCO}^{+}$model spectra are a much better match to the observations, although the western spectrum has a small velocity shift toward the blue. The difference between the eastern and western spectra may be due to the rotation of SFO 11NE SMM1 about its north-south axis, although the difference in the centroids of the two lines is too small $\left(\sim 0.2 \mathrm{~km} \mathrm{~s}^{-1}\right)$ to show conclusive evidence for rotation. The model offers the best fit to the southern spectrum, reproducing the relatively broad red peak, the small possible self-absorption dip and the smaller blue peak visible in the observed spectrum.

The best-fitting model spectra of $\mathrm{HCO}^{+}$and $\mathrm{H}^{13} \mathrm{CO}^{+}$towards the central line-of-sight of SFO 11NE SMM1 are shown in Fig. 2, whilst the model $\mathrm{HCO}^{+}$spectra over the entire observed 5-point grid are shown in Fig. 4. The velocity field that best reproduces the observed $\mathrm{HCO}^{+}$line profiles is of a central core collapsing at a constant velocity of $0.1 \mathrm{~km} \mathrm{~s}^{-1}$ surrounded by an outer envelope expanding at a constant velocity of $0.3 \mathrm{~km} \mathrm{~s}^{-1}$. The central core has a radius of $0.1 \mathrm{pc}$, consisting of the inner eight model shells, and the outer envelope comprises the outer two shells of the model.

It was found that the central $\mathrm{HCO}^{+}$line profile is particularly sensitive to the difference in velocity between the collapsing core and expanding envelope. Changing their relative velocities by only $0.1 \mathrm{~km} \mathrm{~s}^{-1}$ is sufficient to drastically alter the relative brightness of the red and blue peaks of the central 


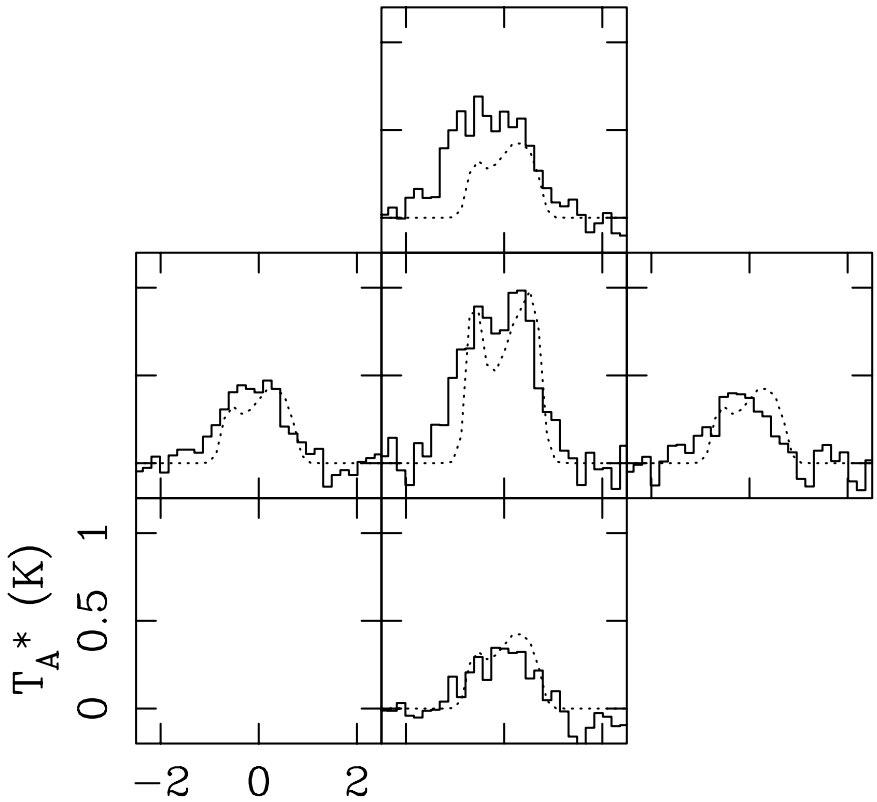

\section{Velocity $(\mathrm{km} / \mathrm{s})$}

Fig. 4. The $\mathrm{HCO}^{+} J=3-2$ grid-map of SFO $11 \mathrm{NE}$ (solid lines) and the best-fitting line model (dotted lines) described in Sect. 3.

spectrum. The central $\mathrm{HCO}^{+}$line profile is also sensitive to the relative diameters of core and envelope. Good fits were obtained for an expanding envelope consisting of the outer three shells of the model, changing the envelope to comprise of the outer two layers or the outer four layers results in red peaks of the $\mathrm{HCO}^{+}$central spectrum that are inconsistent with the observations.

The $\mathrm{HCO}^{+}$line profiles could not be reproduced by assuming a constant $\mathrm{HCO}^{+}$abundance across the model and we found that smoothly increasing the abundance of $\mathrm{HCO}^{+}$ from $10^{-9}$ at the model edge to $5 \times 10^{-8}$ at the model centre gave the best results. The velocity dispersion in the model primarily affects the width of the resulting line profiles. We assumed a constant value of $0.3 \mathrm{~km} \mathrm{~s}^{-1}$ for the velocity dispersion, which closely matches the observed linewidths of the $\mathrm{HCO}^{+}$and $\mathrm{H}^{13} \mathrm{CO}^{+}$lines. The model spectrum at the central position is narrower than the observed spectrum and this could indicate that the velocity dispersion increases towards the centre of the cloud. However, in the interests of restraining the number of free parameters on the model we chose not to explore this possibility.

\section{A collapsing molecular core with an expanding outer envelope?}

In the previous section we described our physical model for SFO 11NE SMM1 as a dense central core of radius $0.1 \mathrm{pc}$ collapsing at $\sim 0.1 \mathrm{~km} \mathrm{~s}^{-1}$, surrounded by an outer envelope expanding at a velocity of $0.3 \mathrm{~km} \mathrm{~s}^{-1}$. Here, we will explore the consequences of and offer a plausible explanation for the collapse and expansion of the molecular cloud core.
Integrating over the entire model results in a total mass of $25 M_{\odot}$ for SFO 11NE SMM1, which is within a factor of 2 of the greybody estimate of Thompson et al. (2004). As the density distribution in the model goes as $r^{-3 / 2}$ and the model shells are logarithmically spaced, most of the mass lies in the outer shells of the model. For our collapsing core and expanding envelope this translates into the core possessing roughly a third of the total mass $\left(8 M_{\odot}\right)$, whilst the envelope consists of the remaining two-thirds $\left(17 M_{\odot}\right)$.

The overall picture of SFO 11NE SMM1 gained from the modelling is of a low-mass central collapsing core surrounded by a relatively high-mass expanding envelope. It is unlikely that the double-peaked line profile is caused by two unrelated gas components at different velocities (e.g. the core and northsouth ridge identified by Thompson et al. 2004) as the optically thin $\mathrm{H}^{13} \mathrm{CO}^{+}$line shows a single-peak at the systemic velocity. A possible explanation for the observed line profile is that the collapse motions result from infall onto one or more prototstars at the centre of the core, whilst the expanding outer layers represent a photoevaporated flow caused by the illumination of the outer layers of the cloud by the nearby O-star.

We may use the masses of each component, their radii and respective collapse and expansion velocities to estimate the mass infall and expansion rates to first order, allowing us to investigate this hypothesis. The simple relation $\dot{M}=M v / r$, relates the mass infall or expansion rate $\dot{M}$ to the mass of the core or envelope $(M)$, the infall or expansion velocity $(v)$ and the outer radius of the core or inner radius of the envelope $(r)$. The infall rate $\dot{M}_{\text {in }}$ estimated from this relation is $\sim 7 \times 10^{-6} M_{\odot} \mathrm{yr}^{-1}$ and the corresponding expansion rate $\dot{M}_{\text {exp }}$ is $\sim 4 \times 10^{-5} M_{\odot} \mathrm{yr}^{-1}$.

The infall rate inferred from the model is consistent with a purely gravitational flow or free-fall collapse, i.e. $\dot{M}_{\text {in }} \simeq \sigma^{3} / G$, where $\sigma$ is the velocity dispersion (predicted to be $0.3 \mathrm{~km} \mathrm{~s}^{-1}$ from the model) and $G$ is the gravitational constant (Williams \& Garland 2002). The infall rate is also approximately equal to that that estimated for a roughly solar-mass protostar (Reid et al. 2002; Zhou et al. 1995). Is it possible that we are observing infall onto a single protostellar object at the centre of SFO 11NE SMM1?

2MASS near-infrared images reveal a candidate cluster of class I protostars and YSOs at the heart of SFO 11NE SMM1 (Thompson et al. 2004) and so it is possible that we are observing gas that is either collapsing onto the cluster as a whole or perhaps onto individual protostars within the cluster. Hydrodynamical models of cluster formation suggest that as the cluster forms and contracts the steepening gravitational potential funnels an accreting gas flow onto the cluster (Bonnell et al. 2001). We note, however, that the mass infall rate within SFO 11NE SMM1 is much lower than has been observed for other protostellar clusters (e.g. NGC 2264 IRS1; Williams \& Garland 2002), where the infall rate can be up to 2 orders of magnitude greater than that for single protostars. For the gas to be undergoing a large scale collapse onto the cluster as a whole there must be a retarding mechanism in operation to slow the infall rate to that consistent with a single protostar.

One of the predictions of radiative-driven implosion (RDI) models of bright-rimmed clouds is the presence of a 
photoevaporated flow from the surface of the cloud illuminated by the nearby OB star (Bertoldi 1989; Lefloch \& Lazareff 1994). The expanding outer layers of the cloud, may in part be due to the presence of a photoevaporated flow from the surface of the cloud, although the large mass $(2 / 3$ of the total molecular cloud core mass) and size of the expanding layer are somewhat difficult to reconcile with a photoevaporated flow alone. The mass loss rate from SFO 11NE SMM1 caused by the photoevaporated flow from the cloud surface was estimated by Thompson et al. (2004) to be approximately 3.5 $M_{\odot} \mathrm{Myr}^{-1}$, which is roughly an order of magnitude lower than that implied by the mass and expansion velocity of the outer envelope $\left(\sim 40 M_{\odot} \mathrm{Myr}^{-1}\right)$.

The relatively high mass expansion rate implies that the envelope expansion is unlikely to be solely driven by a photoevaporative flow. There are two alternative hypotheses that may explain the presence of expanding gas in within SFO 11NE SMM1: either molecular outflow(s) from protostars embedded within SFO 11NE SMM1 or a bulk re-expansion of the bright-rimmed cloud. The former possibility of outflow was mentioned in the previous section: the expanding envelope of the model may be due to one or more molecular outflows mimicking the signature of the bulk expansion of the core.

However, we do not see any evidence for the high-velocity line wings that are the usual indicators of molecular outflow in either the $\mathrm{HCO}^{+}$spectra contained in this paper nor in the CO spectra of Thompson et al. (2004). Although the model of the cloud does not specifially consider a bipolar flow, we would expect the predicted mass of the expanding gas to agree with that contained in a bipolar outflow to at least first order. The mass of outflowing gas predicted by the model $\left(17 M_{\odot}\right)$ is much greater than that typically observed in low mass outflows, which is usually a few $M_{\odot}$ (e.g. Fukui et al. 1993; Richer et al. 2000). It is possible that the mass inferred by the model could be comprised of multiple overlapping flows, particularly in regard of the candidate cluster of protostars and YSOs found at the heart of SFO 11NE SMM1. Although outflows cannot be completely ruled out as an underlying cause of the gas expansion, the lack of supporting evidence for the presence of outflows in this bright-rimmed cloud (for example spatially resolved outflow lobes) and the high outflow masses required to reproduce the model predictions substantially undermine the likelihood of this hypothesis.

Another possibility is that the expanding gas is caused by a bulk re-expansion of the cloud. RDI models predict that this effect follows the maximum compression of the cloud by photoionisation-induced shocks. As the maximum compression is reached, the cloud overshoots its equilibrium state and subsequently expands (Lefloch \& Lazareff 1994). Thereafter the cloud follows an oscillating and decaying cycle of compression and re-expansion until the gas reaches a quasi-static hydrodynamic equilibrium phase as a cometary cloud. Perhaps the expanding outer layers of the model cloud correspond to this expansion phase.

Lefloch \& Lazareff define a "radial momentum" for their RDI model clouds which is the integral of the gas momentum over the cloud volume. The radial momentum of our expanding envelope $\left(0.8 M_{\odot} \mathrm{pc} \mathrm{Myr}^{-1}\right)$ is well within the angular momentum limits evaluated by Lefloch \& Lazareff (1994), even taking into account the relatively high mass fraction of the envelope to central core. Thus it seems that the expanding outer envelope is consistent with the re-expansion phase of the RDI models of bright-rimmed clouds, which is suggested to occur approximately $3 \times 10^{5}$ years after the initial exposure of the cloud to ionising radiation (Lefloch \& Lazareff 1994). This value is consistent with the upper bound on the cloud age derived from the projected distance to the ionisation bound of the HII region (Thompson et al. 2004).

On balance, given the lack of supporting evidence for molecular outflows in SFO 11NE SMM1 we conclude that the most likely cause of the gas expansion is a genuine bulk expansion of the cloud following the maximum compression of the cloud by photoionisation-induced shocks. The likelihood is that we are seeing SFO 11NE SMM1 in the beginning of its re-expansion stage, as this stage is the most consistent with the dynamic age of the associated HII region and the duration that the cloud has been exposed to the UV radiation from the nearby OB star. However, in order to confirm this hypothesis further observations aimed at confirming or denying the presence of bipolar molecular outflow are a high priority.

\section{Summary and conclusions}

We observed the bright-rimmed cloud core SFO 11NE SMM1 in sub-millimetre wave lines of $\mathrm{HCO}^{+}$and $\mathrm{H}^{13} \mathrm{CO}^{+}$to search for the characteristic optically thick blue-peaked infall signature (e.g. Myers et al. 2000). The $\mathrm{HCO}^{+}$emission toward SFO 11NE SMM1 is indeed double-peaked, but with an excess toward the red end of the spectrum rather than the blue. The $\mathrm{H}^{13} \mathrm{CO}^{+}$spectra show a single peak at the systemic velocity and this suggests that the double-peaked optically thick profile originates from a single gas core rather than a line-of-sight coincidence of two different velocity components.

The line data were modelled with the radially symmetric radiative transfer model RATRAN (Hogerheijde \& van der Tak 2000). SCUBA and IRAS HIRES data from Thompson et al. (2004) were used to construct a detailed physical model of the temperature and density structure of the cloud core against which the line emission could be modelled. The resulting physical model of the source is a cloud core of radius $0.26 \mathrm{pc}$, with a density at the outer radius of $4.5 \times 10^{3} \mathrm{~cm}^{-3}$ increasing toward the centre of the core as $r^{-3 / 2}$. The SED and radial flux profile of the core suggest that the temperature of the core consists of two components: an inner warm region at $30 \mathrm{~K}$ surrounded by a colder envelope of $18 \mathrm{~K}$.

The $\mathrm{HCO}^{+}$and $\mathrm{H}^{13} \mathrm{CO}^{+}$line profiles were found to be consistent with a combination of a central core collapsing at a constant velocity of $0.1 \mathrm{~km} \mathrm{~s}^{-1}$, surrounded by an expanding envelope moving outwards at a constant velocity of $0.3 \mathrm{~km} \mathrm{~s}^{-1}$. We derive the mass infall and expansion rates to first order by a simple consideration of the mass, velocity and radius of the collapsing and expanding regions. The mass infall rate is consistent with that estimated for a single solar-mass protostar (e.g. Zhou 1995) and it is possible that we are observing collapse onto a single member of the infrared cluster seen at the heart of SFO 11NE SMM1 (Thompson et al. 2004). 
The expansion rate is an order of magnitude larger than can be sustained by a photoevaporative flow from the outer layers of the cloud (Lefloch \& Lazareff 1994). The expansion is consistent with either the bulk re-expansion of the cloud predicted by the RDI models of Lefloch \& Lazareff (1994) or multiple unresolved bipolar molecular outflows. Given both the lack of supportive evidence for outflows and the relatively high mass of expanding gas predicted by the radiative transfer modelling we conclude that the likeliest scenario for the expanding gas is a bulk re-expansion of the bright-rimmed cloud. If so, SFO 11NE SMM1 is at the beginning of its re-expansion phase following the maximum compression of the cloud by photoionisation-induced shocks.

Although our model of the cloud core as undergoing central collapse and outer expansion is consistent with the data, we stress that this is just one possible interpretation. Observations of higher sensitivity and spatial resolution are required to investigate the kinematics of SFO 11NE SMM1 at smaller spatial scales and provide sufficient data for fully two-dimensional radiative transfer modelling of the line profiles (e.g. Hogerheijde $\&$ van der Tak 2000; Phillips \& Little 2000). In particular, the possibilities that the cloud core is rotating about its north-south axis and that the observed expansion may be in part due to bipolar molecular outflows must be investigated. Our forthcoming survey of a statistically significant number of BRCs will also reveal whether infall motions are as common within these clouds as more isolated low-mass star-forming regions.

Acknowledgements. The authors would like to thank Larry Morgan for struggling through his altitude-induced adversity to assist with the observations, Antonio Chrysostomou for useful discussions, and the JCMT staff for a pleasant and productive observing run. MAT is supported by a PPARC postdoctoral grant.

\section{References}

Bertoldi, F. 1989, ApJ, 346, 735

Bonnell, I. A., Clarke, C. J., Bate, M. R., \& Pringle, J. E. 2001, MNRAS, 324, 573

Elmegreen, B. G. 1991, in The Physics of Star Formation and Early Stellar Evolution, ed. C. J. Lada, \& N. D. Kylafis (Dordrecht: Kluwer), 35
Evans, N. J., II 1999, ARA\&A, 37, 311

Frerking, M. A., Langer, W. D., \& Wilson, R. W. 1982, ApJ, 262, 590

Fukui, Y., Iwata, T., Mizuno, A., Bally, J., \& Lane, A. P. 1993, Protostars and Planets III, 603, ed. E. H. Levy, \& J. I. Lunine (Tucson: Univ. Arizona Press)

Gregersen, E. M., Evans, N. J., Zhou, S., \& Choi, M. 1997, ApJ, 484, 256

Gregersen, E. M., Evans, N. J., Mardones, D., \& Myers, P. C. 2000, ApJ, 533, 440

Hennebelle, P., Whitworth, A. P., Gladwin, P. P., \& André, P. 2003, MNRAS, 340, 870

Hogerheijde, M. R., \& van der Tak, F. F. S. 2000, A\&A, 362, 697

Hogerheijde, M. R., van Dishoeck, E. F., Blake, G. A., \& van Langevelde, H. J. 1998, ApJ, 502, 315

Lada, E. A., Strom, K. M., \& Myers, P. C. 1993, Protostars and Planets III, 245, ed. E. H. Levy, \& J. I. Lunine (Tucson: Univ. Arizona Press)

Lee, C. W., Myers, P. C., \& Tafalla, M. 2001, ApJS, 136, 703

Lee, C. W., Myers, P. C., \& Tafalla, M. 1999, ApJ, 526, 788

Lefloch, B., \& Lazareff, B. 1994, A\&A, 289, 559

Lefloch, B., \& Lazareff, B. 1995, A\&A, 301, 522

Mardones, D., Myers, P. C., Tafalla, M., et al. 1997, ApJ, 489, 719

Myers, P. C., Evans, N. J., \& Ohashi, N. 2000, Protostars and Planets IV, 217, ed. V. Manning, A. P. Boss, \& S. S. Russell (Tucson: Univ. Arizona Press)

Ogura, K., Sugitani, K., \& Pickles, A. 2002, AJ, 123, 2597

Ossenkopf, V., \& Henning, T. 1994, A\&A, 291, 943

Phillips, R. R. \& Little, L. T. 2000, MNRAS, 317, 179

Prestage, R. M., Meyerdierks, H., Lightfoot, J. F., et al. 2000, Starlink User Note 17, Starlink Project, CCLRC

Rawlings, J. M. C., \& Yates, J. A. 2001, MNRAS, 326, 1423

Reid, M. A., Pudritz, R. E., \& Wadsley, J. 2002, ApJ, 570, 231

Richer, J. S., Shepherd, D. S., Cabrit, S., Bachiller, R., \& Churchwell, E. 2000, Protostars and Planets IV, 867, ed. V. Manning, A. P. Boss, \& S. S. Russell (Tucson: Univ. Arizona Press)

Sugitani, K., Fukui, Y., \& Ogura, K. 1991, ApJS, 77, 59

Sugitani, K., \& Ogura, K. 1994, ApJS, 92, 163

Thompson, M. A., White, G. J., Morgan, L. K., et al. 2004, A\&A, 414, 1017

Williams, J. P., \& Myers, P. C. 1999, ApJ, 511, 208

Williams, J. P., \& Garland, C. A. 2002, ApJ, 568, 259

De Vries, C. H., Narayanan, G., \& Snell, R. L. 2002, ApJ, 577, 798

Zhou, S. 1995, ApJ, 442, 685 